\title{
A Note on Finite Groups all of Whose Subgroups are C-Normal
}

\author{
Z. Mostaghim \\ School of Mathematics, Iran University of Science and Technology, Tehran, Iran
}

\begin{abstract}
A subgroup $H$ of a group $G$ is said to be $c$-normal in $G$ if there exists a normal subgroup $N$ of $G$ such that $H N=G$ and $H \cap N \leq \operatorname{Core}(H)$ where $\operatorname{Core}(H)$ is the largest normal subgroup of $G$ contained in $H$. In this paper we consider finite $p$-groups of order at most $p^{4}$ where $p$ is a prime and show that all of their subgroups are $c$-normal. Also we study some classes of finite groups whose all of subgroups are $c$-normal.

Index Terms - $c$-normal subgroups, $p$-groups, maximal class, supersolvable groups.
\end{abstract}

\section{Introduction}

The notion of $c$-normal subgroup was introduced for the first time by Wang $^{l}$. He used the $c$-normality of maximal subgroups to give some conditions for the solvability and supersolvability of a finite group. For example, he showed that $G$ is solvable if and only if $M$ is $c$-normal in $G$ for every maximal subgroup $M$ of $G$. In this paper we consider finite $p$ groups of order at most $p^{4}$ where $p$ is a prime and show that all of their subgroups are $c$-normal. Also we study some classes of finite groups whose all of subgroups are $c$-normal.

Throughout, all groups are assumed to be finite groups. Our terminology and notation is standard, $\operatorname{see}^{2}$.

\section{II . Preliminaries}

In this section, we give some definitions and basic results which are essential in the sequel.

\section{Definition 2.1. ${ }^{1}$}

Let $G$ be a group. We call a subgroup $H$ c-normal in $G$ if there exists a normal subgroup $N$ of $G$ such that $H N=G$ and $H \cap N \leq \operatorname{Core}(H)$

It is clear that a normal subgroup of $G$ is a $c$-normal subgroup of $G$ but the converse is not true.

\section{Definition 2.2. ${ }^{1}$}

We call a group $G$ is c-simple if $G$ has no c-normal subgroup except the identity group 1 and $G$.

We can easily show that $G$ is $c$-simple if and only if $G$ is simple.

\section{Lemma 2.3. ${ }^{1}$}

Let $G$ be a group. Then

(1) If $H$ is normal in $G$, then $H$ is $c$-normal in $G$;

$G$ is $C$-simple if and only if $G$ is simple;

(2) If $H$ is $c$-normal in $G, H \leq K \leq G$, then $H$ is $c$-normal in $K$;

(3) Let $K$ be normal in $G$ and $K \leq H$. Then $H$ is $c$-normal in $G$ if and only if $H / K$ is $C$-normal in $G / K$.

Let $p$ be a prime. Now we will give some properties of non-abelian groups of order $p^{4}$.

Lemma 2.4. ${ }^{3}$

Let $G$ be a finite non-abelian $p$-group of order $p^{4}$. Then

(1) $|Z(G)|=p$ or $p^{2}$;

(2) $\left|G^{\prime}\right| \leq p^{2}$.

\section{Lemma 2.5. ${ }^{3}$}

Let $G$ be a finite non-abelian $p$-group of order $p^{4}$. If $Z(G)$ is cyclic of order $p$, then $G^{\prime}$ has order $p^{2}$. Moreover $Z(G)<G^{\prime}$ and $G / Z(G)$ is not abelian.

\section{Definition 2.6. ${ }^{4}$}

A $p$-group $G$ is said to be a special $p$-group if either $G$ is an elementary abelian $p$-group or we have $\Phi(G)=Z(G)=G^{\prime}$ and $G^{\prime}$ is elementary abelian. If the center of a non-abelian special $p$-group $G$ is cyclic, then $G$ is called extraspecial.

Definition 2.7. ${ }^{4}$

A group of order $p^{n}$ is said to be a group of maximal class if the class of $G$ is $n-1$.

\section{Theorem 2.8. ${ }^{4}$}

The groups $G=D_{2 m}, Q_{2 m}, S D_{2 m}$ have the following properties.

(1) The center $Z(G)$ has order 2 and $G / Z(G) \cong D_{m}$.

(2) The derived group coincides with $\Phi(G)$ and the class of $G$ is $n-1$ where $|G|=2^{n}$.

(3) The group $Q_{2 m}$ contains exactly one element of order 2 .

\section{III . Main Results}

\section{Lemma 3.1.}

Let $G$ be an extraspecial $p$-group. Then every subgroup of order $p$ is $c$-normal.

Proof. It is easy to see that $\left|G^{\prime}\right|=p$. Let $H$ be a subgroup of order. If $H \cap G^{\prime}=G^{\prime}$, then $H \triangleleft G$. By lemma $2.3 H$ is $c$ normal in $G$. If $H \cap G^{\prime}=1$, then there exists a maximal subgroup $M$ such that $H \nsubseteq M$.So $G=H M$ and $H \cap M \leq \operatorname{Core}(H)$. Therefore $H$ is $c$-normal in $G$.

\section{Theorem 3.2.}

Let $G$ be a $p$-group of order at most $p^{4}$. Then all of subgroups of $G$ are -normal.

Proof.

If $G$ be an abelian group, then all of subgroups of $G$ are normal and by lemma 2.3, they are $c$-normal. If $G$ be a nonabelian group of order $p^{3}$, then by lemma 3.1 all of subgroups of order $p$ are $c$-normal. It is easy to see that other subgroups of $G$ are normal. If $G$ be a non-abelian group of order $p^{4}$, then 
by lemmas 2.4 and 2.5 we have two cases:

\section{Case1.}

Let $\left|G^{\prime}\right|=p$, therefore $|Z(G)|=p^{2}$.

Let $H$ be a subgroup of order $p$. If $H \cap G^{\prime} \neq 1$, then $H=G^{\prime}$ and $H$ is a normal subgroup.

Let $H \cap G^{\prime}=1$. If there exists a maximal subgroup $M$ such that $H \nsubseteq M$, then $G=H M$ and $H \cap M \leq \operatorname{Core}(H)$. Therefore $H$ is a $c$-normal subgroup.

Let $H \leq \Phi(G)$, so $\Phi(G)=H G^{\prime}$ and $|\Phi(G)|=p^{2}$. Since $G / Z(G)$ is an elementary abelian group, so $\Phi(G) \leq Z(G)$ and therefore $H$ is a normal subgroup.

Let $H$ be a subgroup of order $p^{2}$. We have $|H \cap Z(G)|=1, p$ or $p^{2}$. If $|H \cap Z(G)|=p^{2}$, then $H=Z(G)$ and $H$ is a normal subgroup. If $|H \cap Z(G)|=1$, then $G=H Z(G)$ and $H \cap Z(G) \leq \operatorname{Core}(H)$. Hence $H$ is $c$-normal in $G$.

Let $|H \cap Z(G)|=p$. It is easy to see that $Z(G)=\Phi(G)$. If $H=\Phi(G)$, then $H$ is a normal subgroup. Otherwise there exists a maximal subgroup $M$ such that $H M=G$ and $|H \cap M|=p$. It is easy to see that $H \cap M \leq \operatorname{Core}(H)$ and therefore $H$ is a $c$-normal subgroup.

Case2.

Let $\left|G^{\prime}\right|=p^{2}$, therefore $|Z(G)|=p$.

Let $H$ be a subgroup of order $p$. If $H \cap Z(G)=H$, then $H$ is a normal subgroup. Let $H \cap Z(G)=1$. If there exists a maximal subgroup $M$ such that $H \cap M=1$, then $H$ is a $c$ normal subgroup. Otherwise $H \leq \Phi(G)=G^{\prime}=H Z(G)$. It is easy to see that $H c h G^{\prime}$ and then $H$ is a normal subgroup.

Let $H$ be a subgroup of order $p^{2}$. Hence $\left|H \cap G^{\prime}\right|=p$ or $p^{2}$.If $\left|H \cap G^{\prime}\right|=p^{2}$, then $H$ is a normal subgroup.

Let $\left|H \cap G^{\prime}\right|=p$. If $H=\Phi(G)$, then $H$ is a normal subgroup. Otherwise, there exists a maximal subgroup $M$ such that $G=H M$ and $|H \cap M|=p$. Since $H \cap G^{\prime} c h G^{\prime}$, therefore $\operatorname{Core}(H)=H \cap G^{\prime}=H \cap M$. Hence $H$ is a $c$-normal subgroup.

\section{Theorem 3.3.}

Let $G=D_{2 n}=\langle a, b| a^{n}=1, b^{2}=1, b a b=a^{-1}>$. Then all of subgroups of $G$ are $c$-normal.

\section{Proof.}

When considered geometrically, $D_{2 n}$ consist of $n$ rotations and $n$ reflections of the regular $n$-gon. The subgroups of $D_{2 n}$ are two types:

(1) Those containing rotations only.

(2) Those containing rotations and reflections.

Let $H$ be a subgroup of $G$. We consider two cases.

\section{Case1.}

Let $H$ has no reflection. Then $H=\left\langle a^{j}\right\rangle$ for $0 \leq j \leq n-1$. Thus by lemma $2.3 H$ is $c$-normal in $G$.

\section{Case2.}

Let $H$ be of type 2 .

(1) Let $a^{j} \notin H$ for $0<j \leq n-1$, so we have $|H|=2$. Now let $N=\langle a\rangle$. Then $N$ is a normal subgroup, $G=H N, H \cap N=1$. Hence $H$ is a $c$-normal subgroup.
(2) Let there exists $i>0$ such that $a^{i} \in H$. Now let $m=\min \left\{i|i\rangle 0, a^{i} \in H\right\}$ and $N=\langle a\rangle$, then $|H|=2 l$, $(1<l \leq n)$ and $H N=G$. Also we have $H \cap N=\left\langle a^{m}\right\rangle$, then $H \cap N \leq \operatorname{Core}(H)$. Hence $H$ is $c$-normal in $G$.

\section{Theorem 3.4.}

Let $G$ be a 2-group of maximal class. Then all of subgroups of $G$ are $c$-normal.

\section{Proof.}

Since $G$ is a 2-group of maximal class, then $G$ is $D_{2^{m}}(m \geq 3), Q_{2^{m}}, S D_{2^{m}}$. We consider three cases.

Case1.

Let $G=D_{2^{m}}(m \geq 3)$. Then by theorem 3.3 all of subgroups of $G$ are $c$-normal.

Case2.

Let $G=Q_{m}$, then by theorem $2.8 G / Z(G) \cong D_{2}^{m-1}$. Let $H$ be a subgroup of $G$ such that $Z(G) \subseteq H$, then by lemma 2.3 $H / Z(G)$ is a $c$-normal subgroup of $G / Z(G)$. By using lemma 2.3 we have $H$ is a $c$-normal subgroup of $G$. If $H$ be a subgroup of $G$ and $Z(G) \$ H$, then $x^{j} \notin H$ for $0<j \leq 2^{m-1}$ so we have $|H|=2$. Now let $N=\langle x\rangle$. Thus $N$ is a normal subgroup of $G$ and $G=H N, H \cap N=1$. Hence $H$ is $c$-normal in $G$.

\section{Corollary 3.5.}

Let $G$ be one of the following groups.

(1) A non-nilpotent finite group that all of proper subgroups are Nilpotent.

(2) A non-abelian finite group that all of proper subgroups are abelian. Then every $p$-Sylow subgroup of $G$ is $c$ normal.

\section{Proof.}

For case (i) we can see $|G|=p^{\alpha} q^{\beta}$, where $p$ and $q$ are distinct primes. Also one of Sylow subgroups of $G$ is cyclic and another is normal. Then every $p$-Sylow subgroup of $G$ is $c$-normal. Case (ii) is similar.

\section{Corollary 3.6.}

Let $G$ be a finite supersolvable group and $p \| G \mid$ where $p$ is the smallest prime divisor of $|G|$. Then $p$-Sylow subgroup of $G$ is $c$-normal.

\section{Proof.}

Let $|G|=p_{1}{ }^{\alpha_{1}} p_{2}{ }^{\alpha_{2}} \cdots p_{n}{ }^{\alpha_{n}}$, where $p_{i}$ are primes such that $p_{1}>p_{2}>\cdots>p_{n} .\left(p=p_{n}\right)$ Let $P_{i}$ be a $p_{i}$-Sylow subgroup of $G$ for $1 \leq i \leq n$, then $P_{1} P_{2} \cdots P_{k}$ is a normal subgroup for all $1 \leq k \leq n$. It is easy to see that $P_{n}$ is $c$-normal in $G$.

\section{GAP Program}

In this section we use $\mathrm{GAP}^{5}$ and give a program for finding c-normal subgroups. By using this program we can find all c-normal subgroups of a finite group with two generations. With a few changes in this program we can find a program for finding c-normal subgroups in a finite group with any number of generations and relations.

F:=FreeGroup("a","b"); 


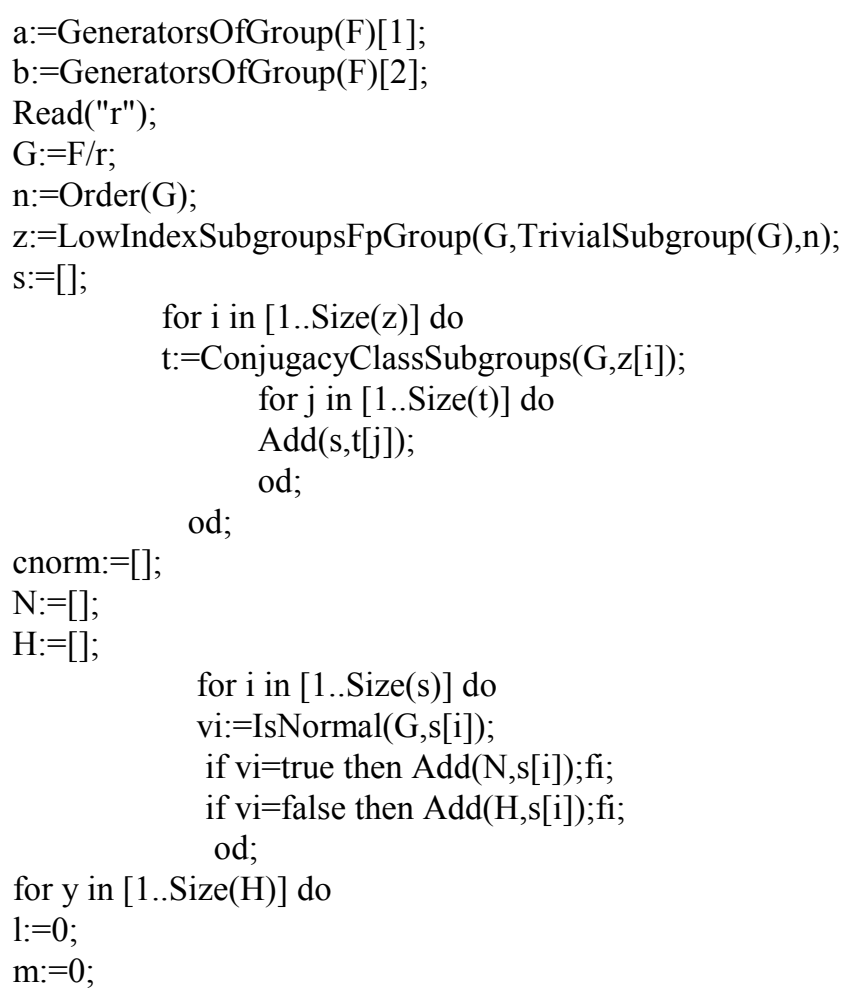

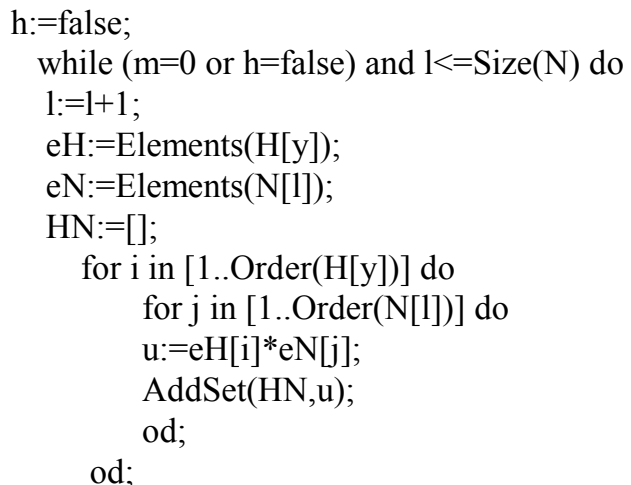

\section{References And Notes}

[1] Y. Wang, J.Algebra 180, (1996).

[2] D. Gorenstein, Finite groups, Chelsea Publishing Company (1980).

[3] H.Behravesh and H.Mousavi ,Proc.Indian Acad.Sci(Math. Sci) 119, (2009).

[4] M. Suzuki, Group Theory II, Springer-Verlag,New York, (1986).

[5] The GAPGroup,GAP-Groups,Algorithms and Programming, (2005). 